BI-TP 99/33

\title{
Numerical evidence for Goldstone-mode effects in the three-dimensional $O(4)$-model ${ }^{*}$
}

\author{
Jürgen Engels and Tereza Mendes ${ }^{\mathrm{a}}$ \\ ${ }^{a}$ Fakultät für Physik, Universität Bielefeld, D-33615 Bielefeld, Germany
}

We investigate the three-dimensional $O(4)$-model on $24^{3}-96^{3}$ lattices as a function of the magnetic field $H$. Below the critical point, in the broken phase, we confirm explicitly the $H^{1 / 2}$ dependence of the magnetization and the corresponding $H^{-1 / 2}$ divergence of the longitudinal susceptibility, which are predicted from the existence of Goldstone modes. At the critical point the magnetization follows the expected finite-size-scaling behavior, with critical exponent $\delta=4.87(1)$.

The continuous symmetry present in the $O(N)$ spin models

$$
\beta \mathcal{H}=-J \sum_{<i, j>} \mathbf{S}_{i} \cdot \mathbf{S}_{j}-\mathbf{H} \cdot \sum_{i} \mathbf{S}_{i}
$$

- where $\mathbf{S}_{i}$ are unit vectors in an $N$-dimensional sphere - gives rise to the so-called spin waves: slowly varying (long-wavelength) spin configurations, whose energy may be arbitrarily close to the ground-state energy. In two dimensions these modes are responsible for the absence of spontaneous magnetization, whereas in $d>2$ they are the Goldstone modes associated with the spontaneous breaking of the rotational symmetry for temperatures below the critical value $T_{c}$. Due to the presence of Goldstone modes, a diverging susceptibility is induced in the limit of small external magnetic field $H$ for all $T<T_{c}$, i.e. everywhere on the coexistence curve (see for example [1]). More precisely: not only the transverse susceptibility, which is directly related to the fluctuation of the transverse (Goldstone) modes, diverges when $H \rightarrow 0$

$$
\chi_{T}\left(T<T_{c}, H\right)=\frac{M(T, H)}{H}=\mathcal{O}\left(H^{-1}\right)
$$

but also the longitudinal susceptibility, given in terms of the magnetization $M$ by $\chi_{L} \equiv$ $\partial M / \partial H$, is predicted to diverge for $2<d \leq 4$. In $d=3$ the predicted divergence is $H^{-1 / 2}$, or

\footnotetext{
*Talk presented by Tereza Mendes
}

equivalently, the behavior for the magnetization will include a "singular" term of order $H^{1 / 2}$

$$
M\left(T<T_{c}, H\right)=M(T, 0)+c H^{1 / 2} .
$$

Indication of this behavior was seen in early numerical simulations of the three-dimensional $O(3)$ model at low temperatures [2].

Here we verify explicitly the predicted singularities - or Goldstone-mode effects - for the three-dimensional $O(4)$ case, by simulating the model in the presence of an external magnetic field and close to the critical temperature $T_{c}$. The low-temperature singularities are still present at $T \lesssim T_{c}$. We are able to compute magnetic critical exponents directly, by varying $H$ at $T_{c}$, and we also use the observed Goldstone-effect behavior to extrapolate our data to $H \rightarrow 0$ and obtain the zero-field critical exponents in very good agreement with the existing values. Using these exponents, we verify finite-size scaling at $T_{c}$. Our motivation for considering the three-dimensional $O(4)$ model is that it is expected to be in the same universality class as QCD at finite temperature for two degenerate quark flavors [3], the magnetic field in the spin model corresponding to the quark mass in QCD.

Our simulations are done using the cluster algorithm. We compute the magnetization along the direction of the magnetic field as

$$
M=\frac{1}{V}<\sum_{i} \mathbf{S}_{i} \cdot \hat{\mathbf{H}}>
$$




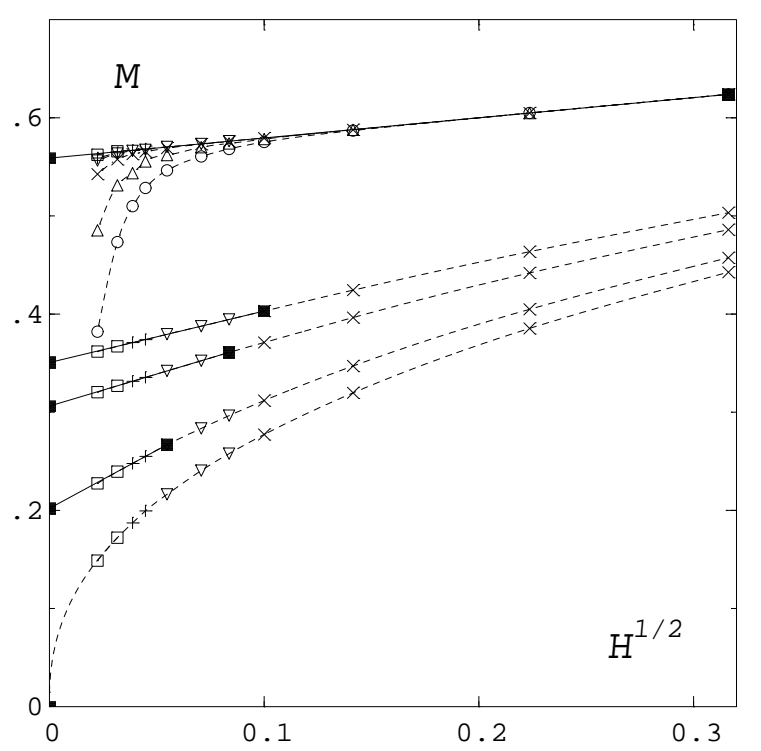

Figure 1. Magnetization for $T \leq T_{c}$.

We note that, due to the presence of a nonzero field, the magnetization defined above is nonzero on finite lattices, contrary to what happens for simulations at $H=0$, where one is led to consider $1 / V<\left|\sum \mathbf{S}_{i}\right|>$, which overestimates the true magnetization.

The susceptibilities are obtained from the spin correlation functions

$$
\begin{aligned}
& G_{L}(x) \equiv<S_{0}^{\|} S_{x}^{\|}>-<S_{0}^{\|}><S_{x}^{\|}> \\
& G_{T}(x) \equiv \frac{1}{3}<\mathbf{S}_{0}^{\perp} \cdot \mathbf{S}_{x}^{\perp}>
\end{aligned}
$$

by $\chi_{L}=\partial M / \partial H=\widetilde{G}_{L}(0) ; \chi_{T}=\widetilde{G}_{T}(0)$. We use for $T_{c}$ the value

$$
1 / T_{c} \equiv J_{c}=0.93590,
$$

obtained in simulations of the zero-field model [4].

In Fig. 1 we show our data for the magnetization for temperatures $T \leq T_{c}$ plotted versus $H^{1 / 2}$. Inverse temperatures are given by $J=J_{c}, 0.95$, $0.98,1.0,1.2$, starting from the lowest curve. We have simulated at increasingly larger values of $L$ at fixed values of $J$ and $H$ in order to eliminate finite-size effects. Except for the curve at $J=1.2$ only these largest-lattice values are shown. Symbols are as explained in Fig. 2.

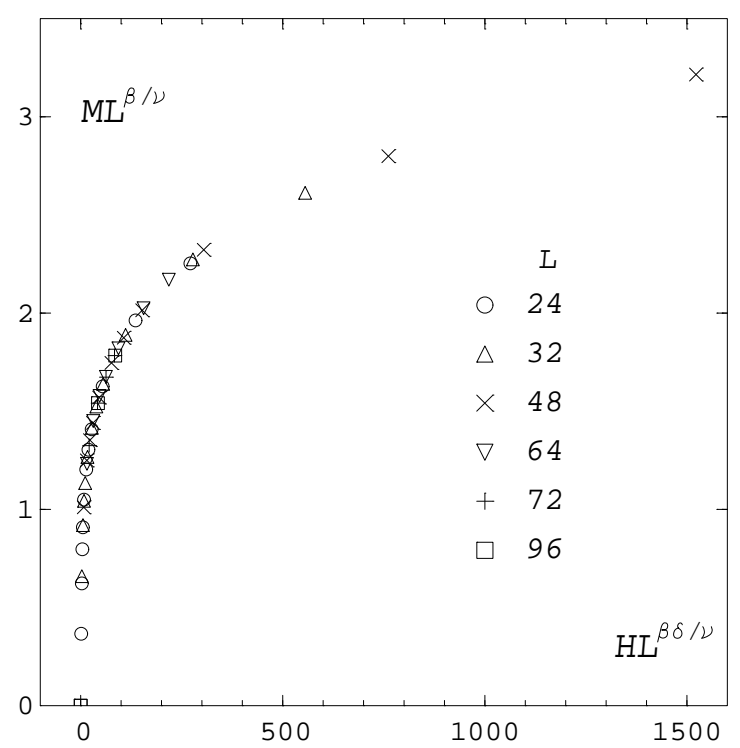

Figure 2. Finite-size-scaling plot for the magnetization at $T_{c}$.

The finite-size effects for small $H$ do not disappear as one moves away from $T_{c}$, but rather increase. Solid lines connect the $y$-axis to the last point (a filled square) included in our fits of the data for small $H$. It can clearly be seen that the predicted behavior (linear in $H^{1 / 2}$ ) holds close to $H=0$ for all temperatures $T<T_{c}$ considered. We thus see evidence that the Goldstonemode effects are observable even very close to $T_{c}$. The straight-line segments become shorter as $T_{c}$ is approached from below, and at $T_{c}$ the magnetization vanishes as a power of $H$, as expected.

We have fitted the data from the largest lattice sizes at $T_{c}$ to the scaling behavior

$$
M\left(T_{c}, H\right) \sim H^{1 / \delta},
$$

obtaining the exponent $\delta=4.87(1)$, in agreement with [4]. The straight-line fits shown in Fig. 1] are used to extrapolate our data to the zero-field limit, and the corresponding zero-field scaling law

$$
M\left(T \lesssim T_{c}, H=0\right) \sim\left(T_{c}-T\right)^{\beta}
$$

yields $\beta=0.38(1)$, also in agreement with [4]. The finite-size effects at $T_{c}$ are very well described by the finite-size-scaling ansatz

$$
M\left(T_{c}, H ; L\right)=L^{-\beta / \nu} Q_{M}\left(H L^{\beta \delta / \nu}\right),
$$




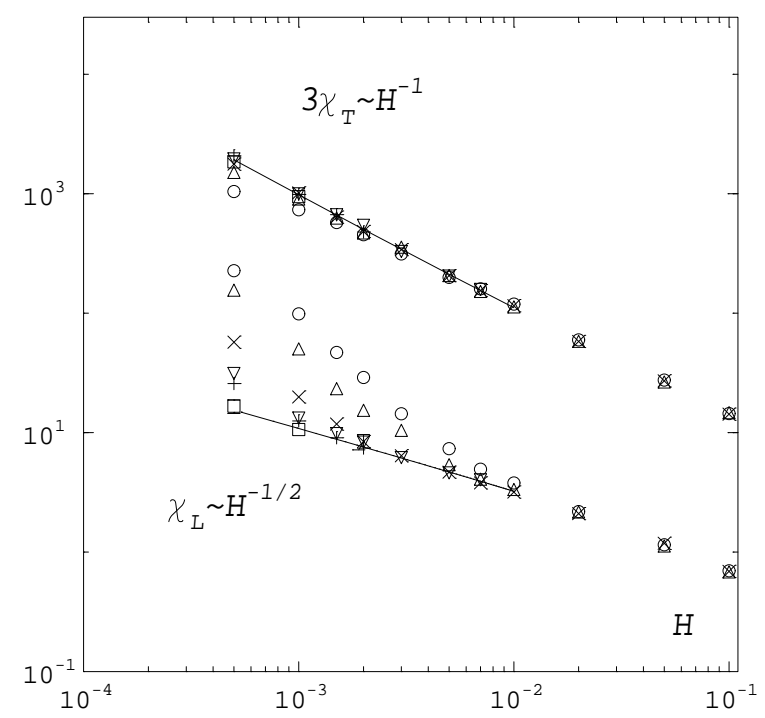

Figure 3. Susceptibilities $\chi_{T}$ and $\chi_{L}$ at low $T$.

as shown in Fig. 2. We see no corrections to finitesize scaling. In Fig. 3 we show a typical behavior for $\chi_{T}$ and $\chi_{L}$ at low temperature, for $J=0.98$. Symbols are as in Fig. 2. The lines $M / H$ (a factor 3 is included for clarity) and $\partial M / \partial H$ (from the fit in Fig. (1) are shown, for comparison respectively with $\chi_{T}$ and $\chi_{L}$. Both lines are obtained from our "infinite-volume" data. Notice the large finitesize effects in $\chi_{L}$.

Finally, we verify numerically the claim in Ref. [1], that the Goldstone-mode singularity at low temperatures is consistent with the magnetic equation of state

$$
(1 / \beta) M^{\delta-1} \chi_{L} \approx c_{1}+c_{2} y^{-1 / 2},
$$

where $y \equiv H / M^{\delta}$. In Figs. 囵 and we show respectively the behavior of $\chi_{L}$ and the combination above for $J=0.95$. We see that the two perturbative predictions are well satisfied at $T \lesssim T_{c}$. Note that the line in Fig. 5 is not obtained using the perturbative coefficients given in [1], but from a fit of our data. This idea of finding nonperturbative coefficients from fits to perturbative forms is very useful in obtaining an expression for the scaling function for this model [5].

This work was supported by the DFG under Grant No. Ka 1198/4-1 and by the TMR-Network ERBFMRX-CT-970122.

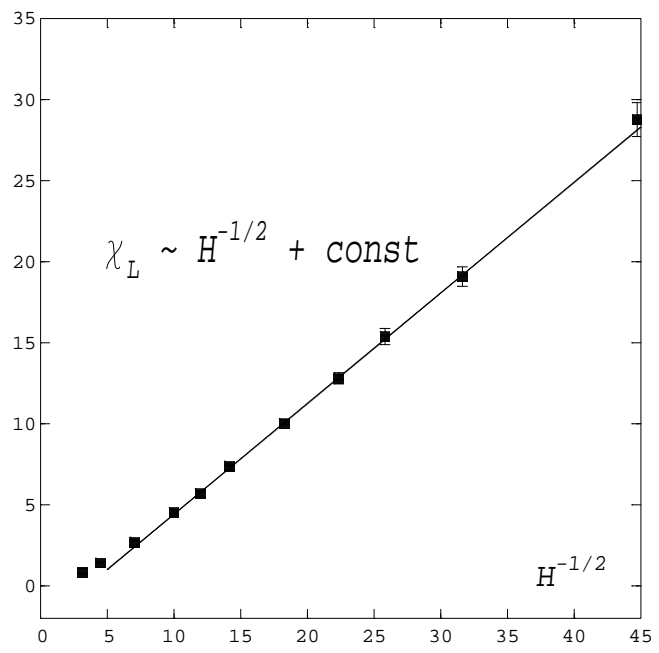

Figure 4 . The susceptibility $\chi_{L}$ at $J=0.95$.

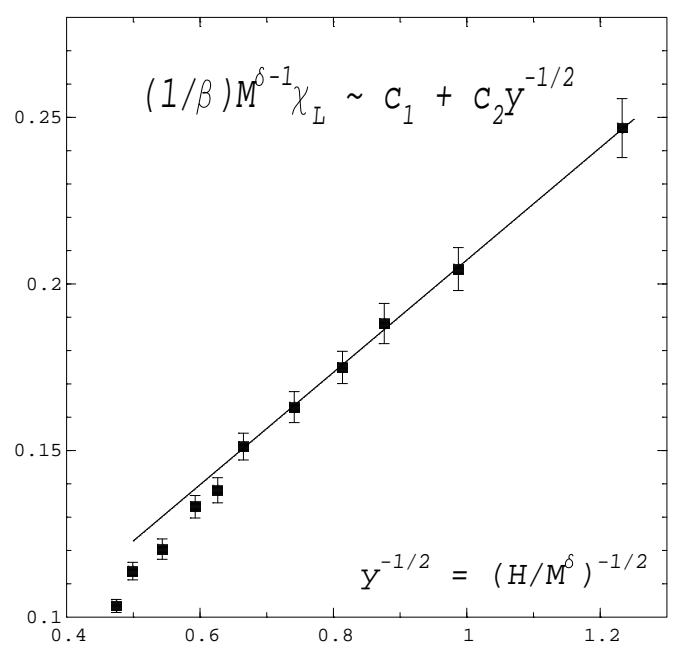

Figure 5. $(1 / \beta) M^{\delta-1} \chi_{L}$ at $J=0.95$.

\section{REFERENCES}

1. D.J. Wallace and R.K.P. Zia, Phys. Rev. B12, 5340 (1975).

2. H. Müller-Krumbhaar, Z. Physik 267, 261 (1974).

3. K. Rajagopal and F. Wilczek, Nucl. Phys. B399, 395 (1993).

4. M. Oevers, diploma thesis, Bielefeld university, 1996; see also K. Kanaya and S. Kaya, Phys. Rev. D51, 2404 (1995).

5. J. Engels and T. Mendes, in preparation. 